\title{
Insectes ravageurs des poissons fumés au cours du stockage et dégâts occasionnés dans la boucle Nord de la Réserve de Biosphère du Dja (Est-Cameroun)
}

\author{
Béranger Raoul TAMGNO ${ }^{1,3 *}$, Hervé TEKOU NGUNTE ${ }^{1}$, Nectaire Lié NYAMSI \\ TCHATCHO $^{1}$, Mama MOUAMFON ${ }^{2}$ et Léonard Simon NGAMO TINKEU ${ }^{3}$ \\ ${ }^{1}$ Laboratoire de Gestion des Ressources Halieutiques, Institut des Sciences Halieutiques de l'Université de \\ Douala, BP 7236, Douala, Cameroun. \\ ${ }^{2}$ Fondation Camerounaise Terre Vivante, BP 12763, Yaoundé, Cameroun. \\ ${ }^{3}$ Unité de Recherches Entomologiques, Faculté des Sciences de l'Université de Ngaoundéré, BP 454, \\ Ngaoundéré, Cameroun. \\ *Auteur correspondant ; E-mail : tamgnoberanger@gmail.com; Tél : (+237) 675577184
}

\section{RESUME}

Dans de nombreux pays en voie de développement, $80 \%$ des captures de poissons sont fumés ou séchés afin de limiter les dégâts. Les insectes constituent un problème récurrent du poisson fumé stocké causant des grandes pertes économiques. Le but de l'étude réalisée dans quatre campements (Djeela, Nemeyong I, Malene I, Ndengué) de la boucle nord de la Réserve de Biosphère du Dja (RBD), était de déterminer la diversité des insectes ravageurs des poissons fumés stockés et leurs dégâts. Dans chaque campement, 25 outils de stockage des poissons fumés ont été inspectés, puis caractérisés et les espèces de poissons fumés identifiées. Lors de cette prospection, des collections de 8 espèces de poissons fumés ont été ramenées et mises en observation au laboratoire. Les insectes émergés des collections ont été dénombrés et identifiés et les dégâts évalués. Il ressort de cette étude que 31 espèces ichtyologiques appartenant à 12 familles sont fumées. Les Cichlidae (35,4\%), les Clariidae $(20,1 \%)$ et les Cyprinidae (10,3\%) sont les familles les plus représentées. Oreochromis niloticus, Clarias gariepinus, Clariallabes manyangae sont les espèces les plus fumées. Les claies sont les outils de stockage les plus utilisés (46,66\%). Excepté C. gariepinus infesté à 80\%, les autres collections sont toutes infestées. Dermestes maculatus, Necrobia rufipes (Coléoptères) et Calliphora sp (Diptères) sont les insectes ravageurs des poissons fumés stockés. D. maculatus est le Coléoptère le plus préjudiciable aux stocks de poissons fumés avec 786 individus $(99,49 \%)$ et $173,8 \mathrm{~g}$ de farine de forage. Les Insectes sont les principaux ravageurs des poissons fumés dans la RBD. C. gariepinus, $C$. manyangae et $M$. moorii sont les poissons fumés les plus attaquées. Les claies sont les outils de stockage les plus répandues.

(C) 2020 International Formulae Group. All rights reserved.

Mots clés : poissons fumés, structure de stockage, insectes ravageurs, dégâts, Réserve de Biosphère du Dja.

\section{Insect pests of smoked fishes during storage and damages done in the north loop of the Dja Biosphere Reserve (East-Cameroon)}

\begin{abstract}
In many developing countries, $80 \%$ of fish catches are smoked or dried to limit losses. Insects are a recurring problem of stored smoked fishes causing great economic losses. The purpose of the study carried out in four camps (Djeela, Nemeyong I, Malene I, Ndengué) of the north loop of the Dja Biosphere Reserve (DBR), was to determine the diversity of insect pests of stored smoked fishes and their damages. In each camp, 25
\end{abstract}


smoked fish storage tools were inspected, then characterized, and the smoked fish species identified. During this survey, collections of 8 species of smoked fishes were brought back and put under observation in the laboratory. The insects emerged from the collections were enumerated and identified and the damage assessed. This study shows that 31 fish species belonging to 12 families are smoked. Cichlids (35.4\%), Clariidae (20.1\%) and Cyprinidae $(10.3 \%)$ are the most common families. Oreochromis niloticus, Clarias gariepinus, Clariallabes manyangae are the most smoked species. Racks are the most used storage tools (46.66\%). With the exception of C. gariepinus infested at $80 \%$, the other collections are all infested. Dermestes maculatus, Necrobia rufipes (Coleopterans) and Calliphora sp (Dipterans) are the insect pests of the stored smocked fishes. D. maculatus is the more prejudicial Coleopteran to the storage of smoked fishes with 786 individuals (99.49\%) and $173.8 \mathrm{~g}$ of drilling flour. Insects are the main pests of smocked fishes in the DBR. C. gariepinus, C. manyangae and M. moorii are the most attacked species. Racks are the most common storage tools.

(C) 2020 International Formulae Group. All rights reserved.

Keywords: Smoked fish, storage structure, insect pests, damages, Dja Biosphere Reserve.

\section{INTRODUCTION}

Les ressources ichtyologiques représentent la principale source de protéine animale pour près de $2 / 3$ de la population mondiale (FAO, 2009, 2012). En Afrique subsaharienne, le poisson couvre en moyenne $22 \%$ des apports en protéines d'origine animale accessibles aux ménages à faibles revenus où le prix de la viande demeure hors de portée du consommateur moyen (FAO, 2016). Le poisson frais est une denrée très périssable dont la durée de conservation dépasse difficilement un jour en milieu tropical du fait du manque d'infrastructures de conservation adéquates et des conditions climatiques (Gram, 2010; Degnon et al., 2013). Pour donc limiter ces pertes, près de $80 \%$ des poissons pêchés sont fumés ou séchés au Nigéria (Folorunso et al., 2006); à Madagascar, en moyenne 5900 tonnes de poissons fumés sont produits par an (Ndrianaivo et al., 2016). En Afrique de l'Ouest, le fumage du poisson est très répandu et se pratique aussi sur les produits de la pêche hauturière (Degnon et al., 2013 ; Sameza et al., 2016). En zone rurale au Cameroun et en fonction des zones agro-écologiques, pratiquement tous les poissons pêchés sont fumés ou séchés. Plus de $80 \%$ du poisson commercialisé en zone rurale est fumé avec des méthodes archaïques (Folorunso et al., 2006).

Du fait de la pratique saisonnière de la pêche dans de nombreuses localités (Contec,
2011), les poissons fumés ou séchés doivent impérativement être stockés pour une consommation ultérieure. La durée de stockage est variable de quelques semaines à plusieurs mois en fonction des saisons, des structures de stockage et du climat. Au cours du stockage, les poissons demeurent toujours hautement périssables et subissent des dépréciations tant quantitatives que qualitatives dues aux proliférations microbiennes et d'infestation par les insectes nuisibles (Keita, 2005 ; Folorunso et al., 2006 ; Ndrianaivo et al., 2016 ; Sameza et al., 2016).

Les pertes post-captures sont estimées à environ 20\%, malgré les efforts que déploient chaque année les femmes pour les limiter en procédant à la conservation du poisson frais par diverses techniques traditionnelles : le fumage, le salage et la fermentation-séchage (Anihouvi et al., 2005). Ces attaques entraînent généralement une perte de revenus et une perte en poissons disponibles pour l'alimentation. Les études menées montrent qu'en Afrique subsaharienne, les dermestes et les nécrobia constituent les insectes les plus nuisibles au stockage du poisson à Madagascar, au Bénin, au Nigéria, au Tchad, au Mali et même au Cameroun (Folorunso et al., 2006). Les pertes peuvent être totales si aucune protection n'est faite (Ndrianaivo et al., 2016). Plusieurs études ont porté sur la lutte antimicrobienne par l'utilisation des huiles essentielles au Bénin, au 
Nigéria et au Cameroun (Folorunso et al., 2006 ; Degnon et al., 2013 ; Sameza et al., 2016; Koudoro et al., 2018). Il n'existe quasiment pas de données récentes sur la lutte contre les insectes ravageurs des stocks de poissons fumés ou séchés au Cameroun et dans la sous-région Afrique Centrale.

Pour contribuer à une gestion durable du poisson fumé stocké, la présente étude se donne pour principal objectif la recherche de la diversité des insectes ravageurs des principales espèces ichtyologiques stockées dans la Réserve de Biosphère du Dja ainsi que les pertes post stockages dans cette région, pas impératif dans la mise au point des bio insecticides.

\section{MATERIEL ET METHODES}

\section{Présentation de la zone d'étude}

La présente étude s'est déroulée dans 4 campements de la Réserve de Biosphère du Dja (RBD) : Djeela $\quad\left(03^{\circ} 21^{\prime}\right.$ N., $\left.012^{\circ} 57^{\prime} \mathrm{E}.\right)$, Nemeyong I $\left(03^{\circ} 21^{\prime}\right.$ N., $012^{\circ} 56^{\prime}$ E. $)$, Malene I ( $03^{\circ} 21^{\prime}$ N., $012^{\circ} 52^{\prime} \mathrm{E}$. $)$

Ndengue $\left(03^{\circ} 20^{\prime} \mathrm{N}\right.$., $013^{\circ} 04^{\prime} \mathrm{E}$.). Cette réserve comprend la Réserve de Faune du Dja et la Zone périphérique. Son altitude varie entre 400 et $800 \mathrm{~m}$.

Sur le plan administratif, la RBD est entre deux régions (Est et Sud) dans un rapport de couverture équivalent à $4 / 5$ et $1 / 5$ respectivement, et deux départements (HautNyong et Dja et Lobo). Elle touche quatre arrondissements dans le département du HautNyong (Est) : Somalomo au Nord, Messamena au Nord-ouest, Mindourou au Nord-Est et Lomié à l'Est Dans le département du Dja et Lobo (Sud), la réserve touche cinq arrondissements: Bengbis au Nord-Ouest, Meyomessala à l'Ouest, Meyomessi, Djoum au Sud et Mintom au Sud-Est. Le climat de la RBD est de type équatorial, avec quatre saisons peu marquées: la grande saison sèche de novembre à mars, la petite saison sèche de juin à juillet, la grande saison de pluies d'août à novembre et la petite saison des pluies de mars à mai.

\section{Etude préliminaire}

Une étude préliminaire a été effectuée dans 17 villages regroupés en 4 secteurs (Maleoleu, Kagnole, Nemeyong et Ekom). Elle a consisté à recenser les différents débarcadères de pêche et d'entrer en contact avec les parties prenantes de la pêche de capture dans la RBD. Compte tenu de la faible capture de certains débarcadères des populations dans les activités de la pêche et des difficultés liées à l'accessibilité dans l'ensemble des campements seuls quatre campements: Malene, Djouo, Djeela et Ndengué ont été finalement prospectés dans le cadre de l'étude (Figure 1).

\section{Détermination de la diversité des espèces de poissons fumés}

L'inventaire des poissons débarqués a été effectué par le suivi des débarquements des pêcheurs (trois jours par semaine dans chaque campement) et par des entretiens semi-dirigés auprès des pêcheurs locaux et des transformateurs. Pour chaque débarquement suivi, les captures étaient évaluées, triées, les noms locaux donnés par les pêcheurs dans la langue locale «le Badjoué ». L'identification des espèces a été faite in situ pour certains spécimens à l'aide des clés d'identification de Mbega et al. (2003) et de Stiassny et al. (2007). Pour des spécimens non identifiés in situ, en plus des noms locaux enregistrés, un spécimen est prélevé, conservé dans de l'alcool à $70 \%$ et étiqueté pour l'identification au laboratoire. Une fois au laboratoire, des observations plus minutieuses sont faites. Auprès des transformateurs et consommateurs, les informations collectées concernaient: noms locaux des espèces fumées, les modules de stockage et les différentes stratégies de limitation des pertes post-stockage des poissons fumés. 
Inspection des structures de stockage et collection in vivo des poissons fumés

Parallèlement à l'identification des poissons frais lors de la prospection, l'inspection de 15 outils de stockage par campement de pêche a été effectuée, soit un total de 60. Durant cette inspection, une grille de caractérisation des structures de stockage combinant le matériau de fabrication, la durée du stockage (court terme, moyen terme, long terme), la portée (ponctuelle, locale, régionale) et le dimensionnement (longueur, largeur, poids) a été utilisée. Au même moment, les éventuels insectes présents dans les structures inspectées étaient collectés, conservés dans de l'alcool à 70\%, puis identifiés au laboratoire. Au cours de cette inspection, des collections in vivo d'un $\mathrm{kg}$ de 8 espèces de poissons les plus fumés ne présentant aucun signe d'infestation initiale y ont été prélevées et ramenées au laboratoire: 5 collections de Clarias gariepinus (Burchell, 1822) «Ntia», 5 de Marcusenius moorii (Gunther, 1867) «Dane», 4 de Enteromus aspilus (Boulenger, 1907) «Akeu», 3 de Paraucheniglanis balayi (Sauvage, 1879) «Mpo'o », 3 de Clariallabes manyangae (Boulenger, 1919) «Mpeh » et 2 collections chacune pour Hemichromis elongatus (Guichenot, 1861) «Sale», Oreochromis niloticus (Linnaeus, 1758) «Boun» et Notoglanidium macrostoma (Pellegrin, 1909) «Boual».

\section{Inventaire et dégâts des insectes nuisibles des poissons fumés stockés}

Une fois au laboratoire, $200 \mathrm{~g}$ de chaque collection ont été pesés et introduits dans des pots en verre de $1200 \mathrm{ml}$ à couvercle ventilé et répété 4 fois. Ces collections ont été mises en observation pendant 4 semaines. Les insectes émergés après cette période ont été collectés, identifiés et comptés. Le taux d'infestation et le nombre d'insectes par gramme de poissons fumés (densité) ont été calculés. La masse de la farine de forage de forage est relevée après avoir tamisé les différentes collections mises en observation. Cette identification a été faite en utilisant la clé d'identification des familles d'insectes de Coléoptères de Freeman (1980), Halstead (1986) et de Delobel et Tran (1993) et par les catalogues d'identification des insectes des produits stockés dans les régions tropicales.

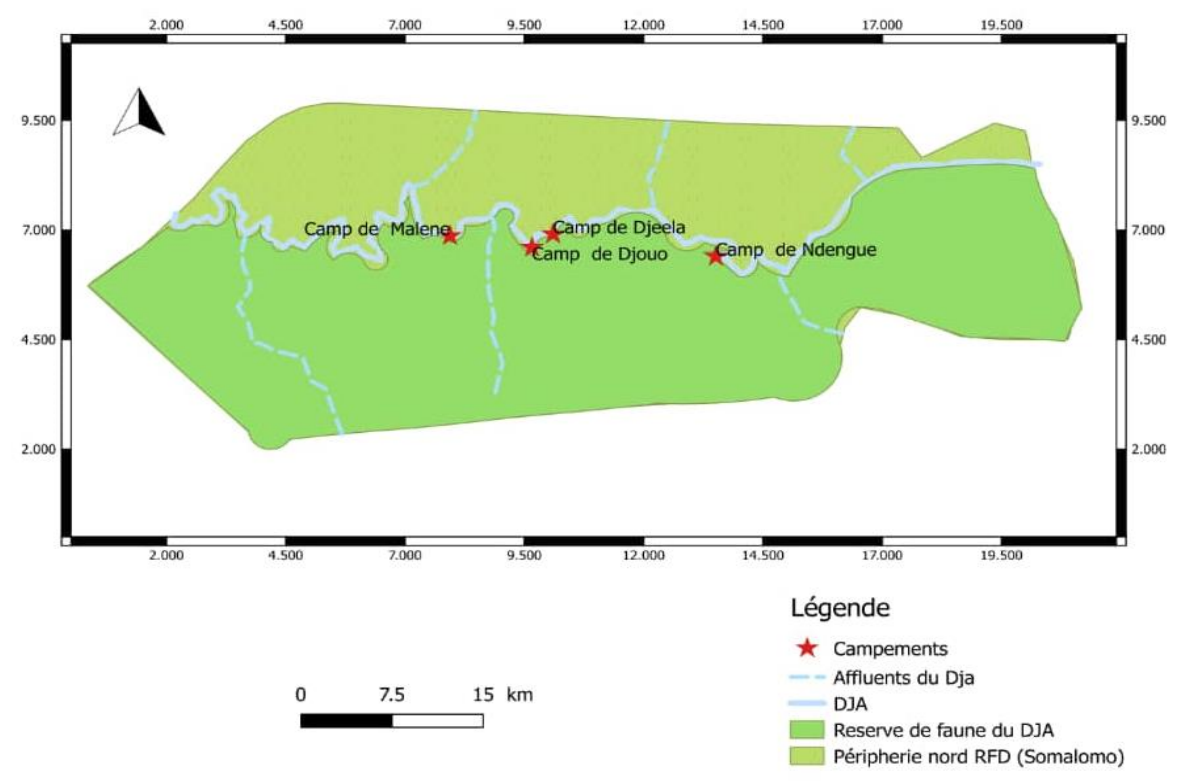

Figure 1: Localisation des campements retenus pour l'étude dans la Réserve de Biosphère du Dja (Est-Cameroun). 


\section{RÉSULTATS}

\section{Diversité des poissons fumés de la Réserve de Biosphère du Dja}

31 espèces appartenant à 12 familles dans l'ensemble des quatre campements ont été inventoriées (Tableau 1). Les familles les plus diversifiées sont celles des Mormyridae et des Cichlidae avec respectivement 6 et 5 espèces. 5 familles sont représentées avec une seule espèce : Hepsetidae, Schilbeidae, Claroteidae, Distichodondidae et Clupeidae. Parmi les familles recensées durant la période de l'étude, les Cichlidés sont les plus représentées $(35,4 \%)$, suivies des Claridae $(20,1 \%)$ et les moins représentées sont Auchenoglanidae $(0,6 \%)$ et Schilbeidae $(0,2 \%)$. Ces deux familles représentent à elles seules plus de 50\% des captures et des poissons fumés, stockés et même commercialisés.

\section{Diversité et caractéristiques des outils de stockage}

Dans le souci de contrecarrer les attaques des Insectes et autres ravageurs, les poissons fumés sont conservés dans divers outils de stockage. Ces structures de stockage sont multiples et variés dans la RBD : claies, paniers, marmites, cartons et sacs en jute (Figure 2). Les claies $(46,66 \%)$ et les paniers (25\%) sont les outils les plus utilisés. Les cartons et les sacs en jute les moins représentés avec respectivement 6 et 5\%. Les claies et les paniers sont en majorité faits de raphia. Les sacs en jute doublés sont de grands sacs pouvant contenir $100 \mathrm{~kg}$ de denrée. L'utilisation de ces outils de stockage est aussi fonction de la durée envisagée du stockage des poissons fumés. Ainsi, les claies, principal outil des transformateurs; sont utilisées pour des stockages de faible durée. Les paniers sont le plus utilisés par les commerçants et sont destinés au stockage dont la durée n'excède pas 14 jours. Les marmites constituent le moyen de stockage des consommateurs et permet de faire des réserves pour des consommations ultérieures et les sacs de jute doublés pour des stockages à longue durée, principalement la période où l'activité de pêche n'est pas propice ou interdite.

\section{Diversité des insectes ravageurs des poissons} fumés de la Réserve de Biosphère du Dja

Les insectes ravageurs du poisson fumé prélevés des structures de stockage et ceux émergés des différentes collections in vivo ramenées de la boucle Nord de la RBD après identification sont de deux ordres: les Coléoptères et les Diptères (Tableau 2). Les Coléoptères appartiennent à deux familles : Cléridés avec l'espèce Necrobia rufipes Degeer et Dermestidés représentée par Dermestes maculatus Degeer. Les Diptères sont représentés par les mouches à viande de la famille des Calliphoridés. L'espèce identifiée est Calliphora sp.

D. maculatus a émergé des 8 collections et $N$. rufipens a émergé de 3 des 8 collections que sont $C$. gariepinus, $C$. manyangae et $B$. aspilus (Tableau 2). D. maculatus et Calliphora $s p$ sont les principaux insectes nuisibles des poissons fumés stockés dans la RBD. C. garipinus, C. manyangae et E. aspilus sont infestées simultanément par $N$. rufipes et D. maculatus.

\section{Niveaux d'infestation des insectes ravageurs des poissons séchés stockés et pertes liées}

Les poissons fumés stockées subissent des attaques d'insectes à des proportions variées (Figure 3, Tableau 3). L'infestation des collections varie selon l'espèce de poisson fumé. Excepté les collections de C. gariepinus infestées à $80 \%$, toutes les autres sont attaquées à $100 \%$. Les insectes ravageurs dans les différentes collections infestées varient avec l'espèce de poissons fumés et aussi du ravageur.

L'importance numérique des insectes extraits des poissons fumés stockés était réalisée par rapport aux nombres d'adultes et de larves d'une part et aussi par rapport à la masse totale des poissons dont ils ont été extraits. C'est ainsi que les dermestes $(99,5 \%)$ sont les insectes les plus importants des stocks avec 771 individus répartis en 11 adultes et 760 larves obtenus des différentes collections 8 semaines après observation. nécrobia ne représente que $0,5 \%$ des coléoptères émergés des collections durant la même période (Tableau 3). M. moorii $(3,31)$ et $P$. balayi 
$(1,64)$ ont les densités les plus élevées et $N$. macrostoma la densité la plus faible $(0,15)$. $D$. maculatus est le principal ravageur et le plus prolifique des poissons fumés stockés dans la zone d'étude (786 individus).

Les masses de farine de forage pesées et les pertes en masse varient selon les différentes collections de poissons fumés (Tableau 4). Les masses de farine de forage oscillent entre $0 \mathrm{~g}$ pour $N$. macrostoma et $59,4 \mathrm{~g}$ chez $C$. gariepinus. 4 collections ont enregistré au bout de 4 semaines d'observation des pertes en masse supérieures à $80 \%$ de leur masse initiale: O. niloticus $(88,89 \%)$ et $M$. moorii $(88,63 \%), C$. manyangae $(86,67 \%)$ et $H$. elongatus $(84,19 \%)$. Ces pertes sont plus du quadruple de celles notées sur la collection de $N$. macrostoma (20\%). Cette dernière collection présente aussi bien la perte en masse la plus faible que la quantité de farine de forage produite par l'activité déprédatrice des insectes ravageurs.

Tableau 1 : Familles et espèces de poissons inventoriées et leur importance dans les différents campements de la Réserve de Biosphère du Dja.

\begin{tabular}{|c|c|c|}
\hline Familles et importance & Noms scientifiques & Noms locaux (Badjoué) \\
\hline \multirow{5}{*}{ Cichlidae $(35,4 \%)$} & Oreochromis niloticus (Linnaeus, 1758) & Boun \\
\hline & Hemichromis bimaculatus Gill, 1862 & Sale \\
\hline & Sarotherodon galilaeus (Linnaeus, 1758) & Boun \\
\hline & Hemichromis elongates (Guichenot, 1861) & Sale \\
\hline & Coptodon zillii (Gervais, 1848) & Bour \\
\hline \multirow{4}{*}{ Alestidae $(10,0 \%)$} & Bryconaethiops microstoma Günther, 1873 & Abom \\
\hline & Brycinus opisthotaenia (Boulenger, 1903) & Nyé demi bande \\
\hline & Brycinus taeniurus (Günther, 1867) & Nyé bande entière \\
\hline & Alestes macrophtalmus (Gunther, 1867) & Sa'a \\
\hline \multirow[t]{2}{*}{ Claroteidae $(6,7 \%)$} & Paraucheniglanis balayi (Sauvage, 1879) & Mpo'o \\
\hline & Paraucheniglanis altipinnis (Boulenger, 1911) & Mvoul \\
\hline \multirow{6}{*}{ Mormyridae $(3,4 \%)$} & Petrocephalus ballayi Sauvage, 1883 & Akuku \\
\hline & Marcusenius moorii (Gunther, 1867) & Dane \\
\hline & Mormyrops angulloides (Linnaeus, 1758) & Zap \\
\hline & Campylomomyrus rhynchophorus (Boulenger, 1898) & Antoa \\
\hline & Isichthys henryi Gill, 1863 & Gue \\
\hline & Mormyrops caballus Pellegrin, 1927 & Epel \\
\hline \multirow{3}{*}{ Mochokidae $(5,6 \%)$} & Atochilus sp & Abaramoko \\
\hline & Synodontis pardalis Boulenger, 1908 & Nke-dja \\
\hline & Synodontis nigrita Valenciennes, 1840 & Elila \\
\hline \multirow[t]{3}{*}{ Clariidae $(20,1 \%)$} & Clarias gariepinus (Burchell, 1822) & Ntia \\
\hline & Clarias pachynema Boulenger, 1903 & Ntia \\
\hline & Clariallabes manyangae (Boulenger, 1919) & Peh \\
\hline \multirow{3}{*}{ Cyprinidae $(10,3 \%)$} & Enteromus aspilus (Boulenger, 1907) & Akeu \\
\hline & Labeo barbatus Boulenger, 1898 & Mpa'a \\
\hline & Labeo parvus (Boulenger, 1902) & Mbogo'o \\
\hline Hepsetidae $(0,9 \%)$ & Hepsetus lineata (Pellegrin, 1926) & Djassiel \\
\hline Schilbeidae $(0,2 \%)$ & Schilbe multitaeniatus (Pellegrin, 1913) & Ntoulou \\
\hline Auchenoglanidae $(0,6 \%)$ & Notoglanidium macrostoma (Pellegrin, 1909) & Boual \\
\hline Distichodondidae $(4,4 \%)$ & Distichodus fasciolatus Boulenger, 1898 & Abackmobi \\
\hline Clupeidae $(2,5 \%)$ & Pellonula leonensis Boulenger, 1916 & Impassa \\
\hline
\end{tabular}




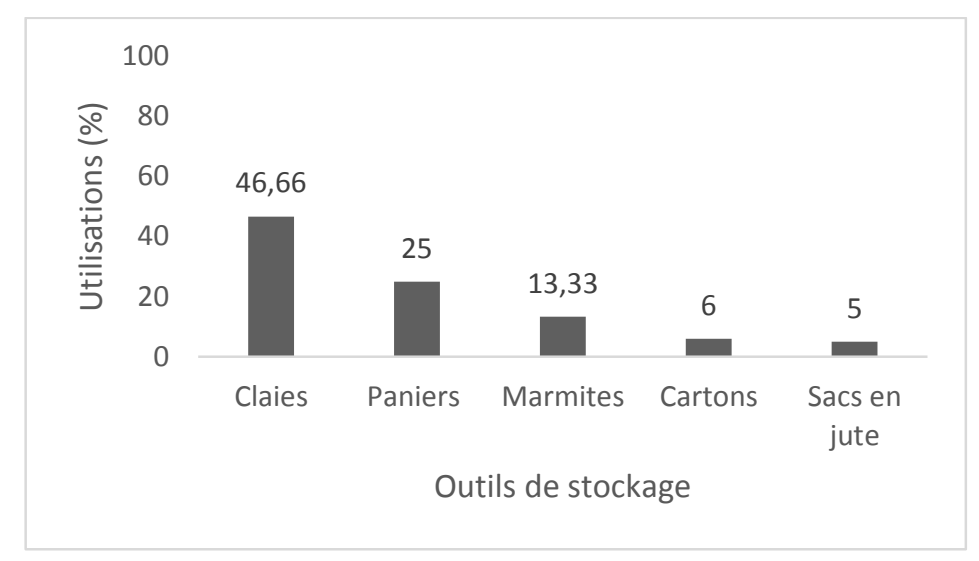

Figure 2 : Fréquence des outils de stockage du poisson fumé utilisés par les populations de la Réserve de Biosphère du Dja.

Tableau 2 : Insectes ravageurs prélevés des différentes collections de poissons fumés.

\begin{tabular}{llll}
\hline Ordres & Familles & Espèces & Espèces de Poissons fumés \\
\cline { 2 - 4 } Coléoptères & Cléridés & $\begin{array}{l}\text { Necrobia } \\
\text { rufipes }\end{array}$ & $\begin{array}{l}\text { Clarias gariepinus, Clariallabes manyangae, } \\
\text { Enteromus aspilus }\end{array}$ \\
\cline { 2 - 5 } & Dermestidés & $\begin{array}{l}\text { Dermestes } \\
\text { maculatus }\end{array}$ & $\begin{array}{l}\text { Clarias gariepinus, Marcusenius moorii, Enteromus } \\
\text { aspilus, Paraucheniglanis balayi, Clariallabes } \\
\text { manyangae, Hemichromis elongatus, Oreochromis } \\
\text { niloticus, Notoglanidium macrostoma }\end{array}$ \\
\hline Diptères & Calliphoridés & Calliphora sp & $\begin{array}{l}\text { Présents dans tous les poissons fumés stockés de la } \\
\text { boucle Nord de la RBD }\end{array}$ \\
\hline
\end{tabular}

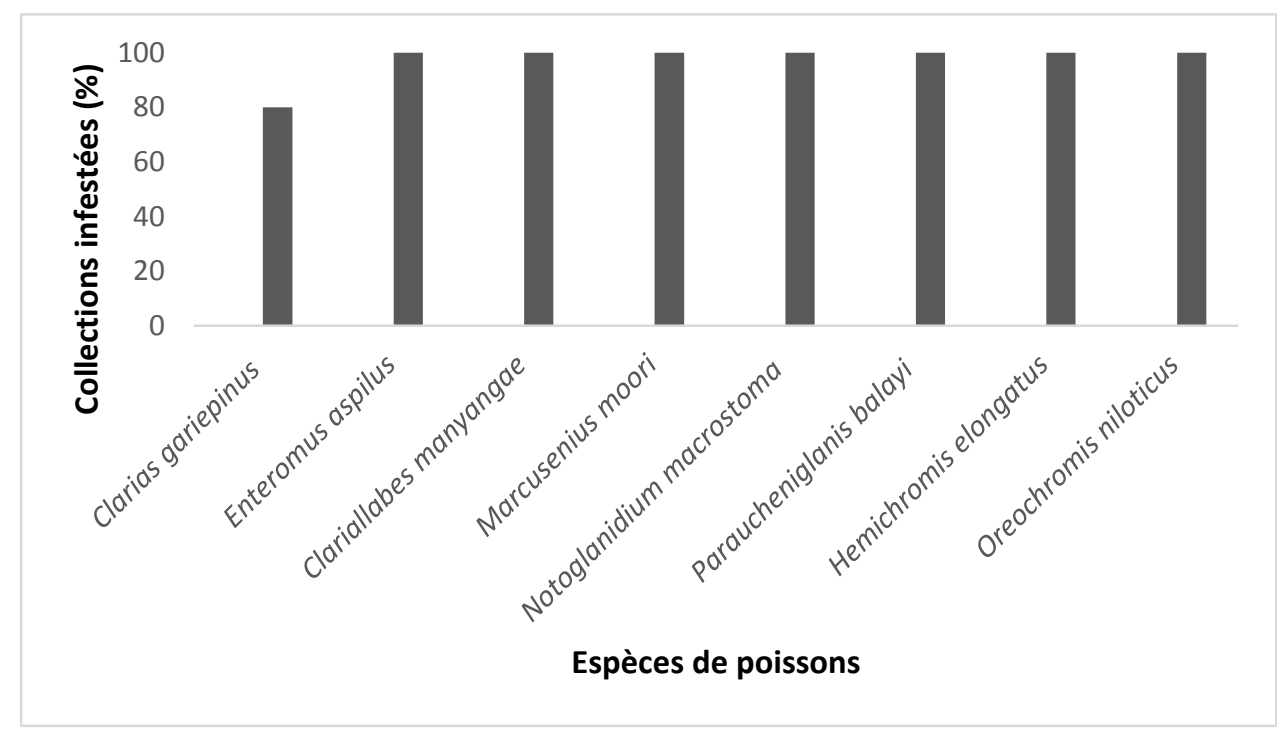

Figure 3 : Taux d'infestation naturelle des collections in vivo de poissons fumés de la Réserve de Biosphère du Dja. 
Tableau 3 : Diversité et importance numérique des insectes extraits des différentes collections de poissons fumés et observés en laboratoire durant 4 semaines.

\begin{tabular}{lllll}
\hline Collections de poissons fumés & Insectes ravageurs & Densité & Imagos & Larves \\
\hline Enteromus aspilus & Dermestes maculatus & 1,1 & 0 & 20 \\
& Necrobia rufipes & & 2 & 0 \\
Clariallabes manyangae & Dermestes maculatus & 1,28 & 0 & 173 \\
& Necrobia rufipes & & 1 & 0 \\
Marcusenius moorii & Dermestes maculatus & 3,31 & 0 & 222 \\
Notoglanidium macrostoma & Necrobia rufipes & & 0 & 0 \\
& Dermestes maculatus & 0,15 & 1 & 1 \\
Paraucheniglanis balayi & Necrobia rufipes & & 0 & 0 \\
& Dermestes maculatus & 1,64 & 3 & 71 \\
Hemichromis elongatus & Necrobia rufipes & & 0 & 0 \\
& Dermestes maculatus & 0,71 & 0 & 35 \\
Oreochromis niloticus & Necrobia rufipes & & 0 & 0 \\
& Dermestes maculatus & 0,71 & 0 & 47 \\
Clarias gariepinus & Necrobiarufipes & & 0 & 0 \\
& Dermestes maculatus & 0,95 & 7 & 206 \\
& Necrobia rufipes & & 1 & 0 \\
\hline Total & & & & 790
\end{tabular}

\section{DISCUSSION}

La diversité ichtyologique inventoriée dans la boucle nord de la Réserve de Biosphère du Dja (Tableau 1) est différente de celle obtenue dans sa partie Sud. En effet, l'étude d'impact environnemental et social réalisée dans la partie Sud a permis de recenser 46 espèces réparties en 20 familles (Claroteidae, Cyprinidae, Mormyridae, Mochokidae, Characidae...) (Contec, 2011). Kharim (2015), toujours dans la partie du sud de la réserve a inventorié 4 ans après 14 familles ichtyologiques. Les différences observées sur la diversité ichtyologique des trois études s'expliqueraient par la méthode de collecte. En effet, l'inventaire des poissons a été fait par observations directes des filets posés, des captures au niveau des pirogues, des débarcadères ainsi que des marchés de Mékin (Contec, 2011). Cependant, toutes les espèces identifiées au cours de cette étude sont connues comme appartenant au bassin du Congo.

La diversité des outils de stockage du poisson fumé développés par les populations trahit les difficultés qu'elles ont à résoudre le problème de stockage dans la boucle Nord de la RBD (Figure 2). En effet, Keita (2005) signalait déjà que le stockage, le conditionnement et le transport font partie de la phase des pertes plus importantes des poissons traités au Mali. Au vue de ces pertes, des auteurs s'accommodent sur le fait que le 
stockage est réussi si à son terme, la denrée n'enregistre de perte significative tant quantitative que qualitative (CIRAD, 2002). Les travaux de Knockaert (1990) ont présenté aussi une diversité des modules de stockage de poissons constitués principalement des paniers de limbes de feuilles tressés recouverts d'un sac en toile de jute ou en plastique, des sacs en toile ou en jute cousus, des cartons ou des caisses fermés avec des cordes. L'avantage de ces outils traditionnels se trouve dans sa meilleure conservation. Le stockage du poisson fumé doit s'effectuer à l'abri de la pluie et du soleil et limiter toute formation éventuelle de rosée à la surface du poisson pendant la nuit. Ces conditions atmosphériques (soleil, pluie, rosée) favorisent le développement des levures et moisissures dans le poisson fumé. C'est ainsi que les ravageurs principalement les insectes continuent à occasionner des pertes aux denrées stockées. Par conséquent, l'emballage idéal pour un stockage en région humide comme la RFD doit être imperméable à l'air, à l'eau et résistant à l'attaque des insectes.

Les dermestes, les nécrobia et les mouches à viande sont les principaux insectes ravageurs du poisson fumé dans la boucle Nord de la Réserve de Biosphère du Dja (Tableau 2). La présence de ses insectes ravageurs n'est pas surprenante. En effet, l'Afrique subsaharienne qui fait partie des zones chaudes du globe présente les conditions climatiques optimales pour la prolifération de ces insectes ravageurs. Les dermestes et les nécrobia constituent les insectes les plus nuisibles au stockage du poisson à Madagascar, au Bénin, au Nigéria, au Tchad, au Mali et même au Cameroun (Delobel et Tran, 1993 ; Keita, 2005 ; Folorunso et al., 2006).

D. maculatus, présente dans toutes les collections de poissons fumés avec des intensités variantes largement supérieures à celles de N. rufipes (Tableau 3) trouverait son explication dans l'origine même des poissons. En effet, les 31 espèces inventoriées lors de cette étude sont des espèces dulcicoles (Stiassny et al., 2007) et D. maculatus est le principal ravageur des poissons traités d'eau douce et $N$. rufipes celui des poissons de mer ou des poissons salés fumés (Ndrianaivo et al., 2016). Une teneur élevée en sel conduit à la mortalité des dermestes ; par contre, c'est dans les poissons salés que nécrobia s'épanouit mieux (Ndrianaivo et al., 2016). La présence de $N$. rufipes dans ces stocks serait accidentelle; en effet, ce ravageur est un grand voilier dont l'habitat qui lui convient est analogue à celui des espèces tropicales de Dermestes dont il peut se nourrir de leurs larves et œufs.

$\mathrm{La}$ forte infestation naturelle des collections de poissons fumés et l'importance numérique surtout des dermestes comme nuisibles prépondérants (Figure 3, Tableau 3) dans la zone d'étude laissent présager des pertes si aucune mesure de protection n'est prise (Tableau 4). En effet, la nutrition des larves et des adultes provoque des pertes quantitatives considérables de poisson séché, ainsi que l'émiettement (FAO, 1996; Ndrianaivo et al., 2016). La perte de qualité peut aussi être due à la présence de corps et de dépouilles d'insectes. L'importance et la valeur des pertes quantitatives causées au poisson séché ont été évaluées par différents chercheurs et les estimations vont d'un chiffre insignifiant jusqu'à $50 \%$ de perte de poids, selon la durée de l'entreposage, la teneur en sel, l'humidité, les conditions climatiques et les conditions générales d'hygiène pendant le traitement et le stockage (Folorunso et al., 2006 ; Degnon et al., 2013 ; Ndrianaivo et al., 2016). Les insectes creusent des galeries dans les poissons et se nourrissent de leur chair, le poisson a tendance à se fragmenter facilement et la friabilité du poisson associée aux odeurs d'altérations rendent le poisson moins attrayant et donc moins appétissant (Ndrianaivo et al., 2016).

\section{Conclusion}

La production halieutique est saisonnière dans de nombreux plans d'eau avec pour conséquence directe la transformation et le stockage des poissons traités destinés à une consommation ultérieure. Les Cichlidés et les 
Clariidés représentent plus de 50\% des poissons fumés dans la boucle nord de la Réserve de Biosphère du Dja. Les espèces les plus fumées sont $O$. niloticus, $C$. gariepinus et C. manyangae. Deux ordres infestent les poissons dans la RBD : Coléoptères et Diptères. Le principal insecte ravageur des poissons fumés est $D$. maculatus. Ce ravageur constitue $95,5 \%$ des insectes ravageurs émergés des stocks. On a observé des pertes quantitatives et qualitatives dues aux dégâts des déprédateurs pendant les analyses effectuées. Si rien n'est fait, une dégradation exponentielle de ces poissons fumés réduira ainsi leurs valeurs économiques. Pour limiter les pertes de la denrée dues aux insectes, il est urgent et nécessaire de promouvoir des outils de protection des poissons traités qui respectent les consommateurs et l'environnement.

\section{CONFLIT D'INTERETS}

Les auteurs déclarent qu'il n'y a aucun conflit d'intérêts.

\section{CONTRIBUTIONS DES AUTEURS}

HTN a mené les travaux sur le terrain et au laboratoire. Il était accompagné par MM qui a financé les campagnes de collecte. NLNT a participé à la rédaction de ce manuscrit. BRT et LSNT sont les porteurs de ce projet, ils ont suivi de bout en bout les activités et ont participé activement à la rédaction de ce manuscrit.

\section{REMERCIEMENTS}

Les auteurs remercient l'International Foundation for Science.

\section{REFERENCES}

Anihouvi VB, Hounhouigan JD, Ayenor GS. 2005. Production et commercialisation du «lanhouin», un condiment à base de poisson fermenté du golf du Bénin. Cah. Agr., 14(3) : 323-330

CIRAD. 2002. Mémento de l'Agronome: Ministère des Affaires Etrangères. France.
Contec. 2011. Rapport final de l'Etude d'impact Environnemental et Social de la centrale hydroélectrique de Mekin et la ligne électrique HT $110 \mathrm{KV}$ reliant la centrale du Réseau Interconnecté Sud (RIS), Département de Dja et Lobo, Région du Sud.

Degnon RG, Faton AN, Adjou ES, Tchobo FP, Dahouenon-Ahoussi E, Soumanou MM, Sohounhloue DCK. 2013. Efficacité comparée des huiles essentielles de deux plantes aromatiques dans la conservation post-fumage du Chinchard (Trachurus trachurus). J. Anim. Plant Sci., 19 (1): 2831-2839.

DOI: http://www.m.elewa.org/JAPS.

Delobel A, Tran M. 1993. Les Coléoptères des Denrées Alimentaires Entreposées dans les Régions Chaudes. ORSTOM/CTA (Faune tropicale XXXII) : France ; $424 \mathrm{p}$.

FAO. 2009. Document technique sur les pêches et l'aquaculture. Rome, $535 \mathrm{p}$.

FAO. 2012. Situation mondiale des pêches et de l'aquaculture. Rome, $241 \mathrm{p}$.

FAO. 2016. La situation mondiale des pêches et de l'aquaculture. Contribuer à la sécurité alimentaire et à la nutrition de tous. Rome. 224 p.

Folorunso AA, Sambo BA, Danjuma M, Usman, A. Ibeawuchi RK, Edosa O. 2006. Effect of insect infestation on nutritional quality of smoked fish species in Jos, Nigeria. Cam. J. Exp. Biol., 2 (1): 26-30.

Freeman P. 1980. Common Insect Pests of Stored Food Products: A Guide to their Identification. British Museum (Natural History): London; 69 p.

Gram L. 2010. Microbiological spoilage of fish and seafood products. In Compendium of the Microbiological Spoilage f foods and Beverages, Sperson WH \& Dolye MP (eds); 87-119.

Halstead DGH. 1986. Keys for the identification of beetles associated with stored products. Introduction and key to 
families. J. Stored Prod. Res., 22(4):163203.

Kéita DMO. 2005. Contribution à l'étude de la qualité des poissons transformés (fumés, séchés) à Bamako, Mopti, Niono et Sélingué. Thèse de doctorat, Université de Bamako, Mali.

Kharim M. 2015. Valorisation des espèces ichtyologiques capturées dans le fleuve Dja. Mémoire de stage d'insertion professionnelle. Master professionnel, Université de Douala, Cameroun.

Knockaert C. 1990. Le Fumage du Poisson (Valorisation des Produits de la Mer). Ifremer, $154 \mathrm{p}$.

Koudoro AY, Konfo CTR, Sessou P, Sohounhloué DCK. 2018. Status of research results in chemistry of biologically active substances in Benin. Int. J. Phytomed., 10(3): 125-136. DOI: 10.5138/0975085.2251.

Mbega J-D, Teugels GG. 2003. Guide de Détermination des Poissons du Bassin Inférieur de l'Ogooué. Presse Universitaire de NAMUR, $165 \mathrm{p}$.
Ndrianaivo EN, Cornet J, Cardi M, Razanamparany L, Berge JP. 2016. Stockage des poissons fumés et ou séchés: cas de Oreochromis niloticus "Fiha saly" malgache. Af. Sci., 12(2) : 254- 265. DOI : http://www.afriquescience.info.

Sameza ML, Tchameni SN, Ekoue JDA, Jazet PMD, Tchoumbougnang F. 2016. Growth inhibition of the stored fish (Ethmalosa frimbiata) fungus Aspergillus flavus, exposed to extracted essential oils from Callistemon citrinus and Ocimum canum. Af. J. Microbiol. Res., 10(30): 1164-1172. DOI: 10.5897/AJMR2016.8028

Stiassny M, Teugels GG, Hopkins C. 2007. Poissons d'Eaux Douces et Saumâtres de Basse Guinée, Ouest de l'Afrique Centrale. IRD éditions, Collection Faune et Flore tropicales $42(1,2)$ : France ; 805 $\mathrm{p}$ et $622 \mathrm{p}$. 